Check for updates

Cite this: RSC Adv., 2018, 8, 25065

Received 15th May 2018

Accepted 5th July 2018

DOI: $10.1039 / c 8 r a 04133 d$

rsc.li/rsc-advances

\section{Design of diverse nanostructures by hydrothermal and microemulsion routes for electrochemical water splitting}

\begin{abstract}
Anirban Das and Ashok Kumar Ganguli (iD *
Hydrothermal and microemulsion methods are low temperature methods used to obtain nanostructures of definite morphologies, sizes, facet termination and other structural features which result in the corresponding unique response to chemical, electrochemical or photochemical stimuli. An efficient catalyst to electrochemically split water to produce hydrogen and oxygen is of scientific, economic and societal relevance, especially due to the abundance of the starting material, water, and due to the product hydrogen, which is an ideal fuel, due to its highest mass density and clean combustion in air. In this review we focus on the hydrogen evolution reaction, HER, and the oxygen evolution reaction, OER, activity of the electrocatalysts produced by hydrothermal or microemulsion methods. The variation in electrochemical response resulting from the unique shape, composition and nano-architecture is discussed. Broadly, the catalysts were categorized as binary and ternary metal alloys as well as metal chalcogenides and oxides. This compilation would aid in the design of more effective water splitting electrocatalysts as well as in the selection of appropriate candidates for advanced mechanistic studies.
\end{abstract}

\section{Introduction}

Fabrication of novel nanomaterials with programmable organization of nanoscale building blocks is a fundamental vision of nanotechnology. These functional nanomaterials show shape

Department of Chemistry, Indian Institute of Technology, Hauz Khas, New Delhi 110016, India. E-mail: ashokganguliiitd@gmail.com; ashok@chemistry.iitd.ernet.ac.in and size dependent properties, ${ }^{1-4}$ thus it is imperative to develop synthetic methodologies which can precisely control the structural details of nanomaterials. Hydrothermal and microemulsion methods are two very useful techniques as these allow directed syntheses of nano-sized materials. The directed syntheses aid in obtaining definite geometries, surface termination, crystallinity, and new nanoscale physical properties which in turn influence the chemical properties and thus

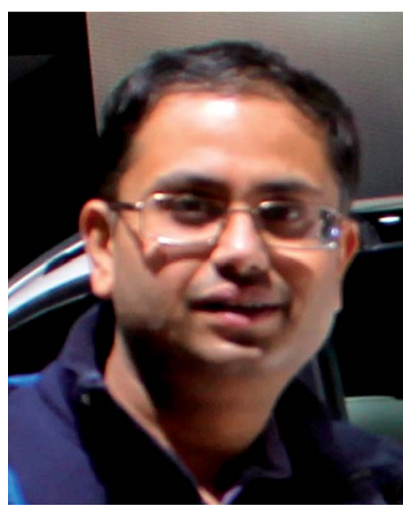

Dr Anirban Das obtained a BSc (Chemistry Honours) degree from Delhi University, India, an $M S$ (Chemistry) from the University of Toledo, Ohio (Prof. Mark Mason, 2007) and a PhD (Inorganic Chemistry) from the University of Idaho (Prof. Chien Wai, 2012). Thereafter, he held postdoctoral positions at Northwestern University (Prof. Eric Weitz, 2012-2014), University of California (Prof. Francisco Zaera, 2014-2015) and at the Wayne State University (Prof. Eranda Nikolla 2015-2017). Currently he is a CSIR Pool Scientist at IIT Delhi, India working with Prof. Ashok Kumar. Ganguli. His current interests include development of photo and electrocatalysts for water splitting applications.

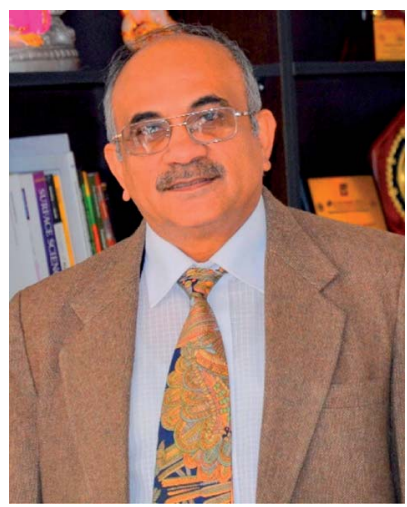

Dr Ganguli is a Professor of Chemistry at IIT Delhi and also the founding Director of the Institute of Nano Science and Technology in Mohali, Punjab. His areas of interest are in the design of nanostructured materials for several applications and also high temperature superconductivity. He has published more than 250 papers and filed five patents. $\mathrm{He}$ is a recipient of several awards including the National Award of Nano Science and Nanotechnology given by DST, Government of India and also a fellow of various science academies. Dr Ganguli is very passionate for promoting education in schools and colleges in underprivileged areas. 


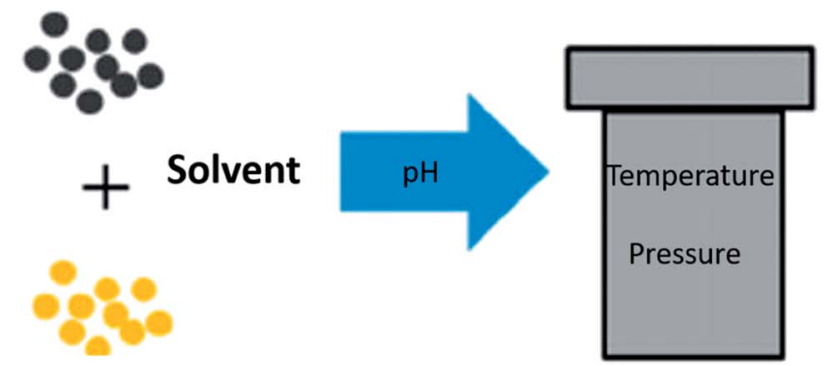

Precursors
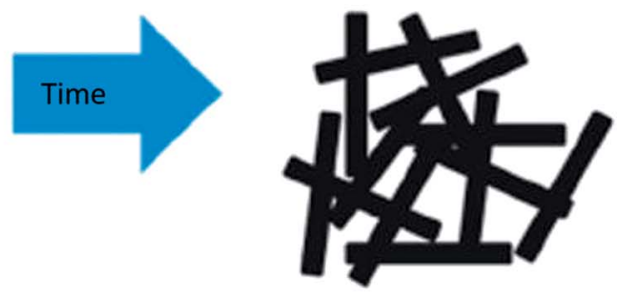

Nanostructures

Scheme 1 The general process involved in a hydrothermal synthesis.

catalytic performance. The main advantage of the hydrothermal method is that it allows the synthesis at lower temperatures than the traditional high temperature routes and at times allows stabilization of metastable phases. It allows for direct crystallization of multi-element oxides without the need for annealing at elevated temperature to induce crystallization. ${ }^{5-8}$ The general process involved in a hydrothermal synthesis is depicted in Scheme 1. Microemulsions containing reverse micelles can be used effectively for the synthesis of nanoparticles of controlled size and structure, where the synthesis of nanoparticles occurs inside the volume defined by water droplets. Reverse micelles have been used for the synthesis of a variety of nanoparticles including metal nanoparticles, alloys, semiconducting oxides, and chalcogenides to exploit among others their optical, magnetic and catalytic properties. ${ }^{9-12}$ The shape of the nanoparticles inside the reverse micellar system can be altered by modulating the parameters affecting (a) film rigidity of reverse micelles and (b) kinetics of crystal growth in the constrained environment. The reverse micellar film rigidity can be controlled ${ }^{13,14}$ by the following parameters: (i) water-tosurfactant molar ratio $\left(W_{\mathrm{x}}\right)$, (ii) intermicellar exchange rate, (iii) nature of the bulk organic solvent, (iv) nature of the surfactant and co-surfactant, and (v) packing parameter. The general process involved in a reverse microemulsion synthesis is depicted in Scheme $2 .{ }^{15}$ Our group has been working extensively for more than a decade, to understand the role of various parameters of microemulsions which matter in the tailored synthesis of nanostructures. ${ }^{16-21}$

\section{(a) \\ (b)}

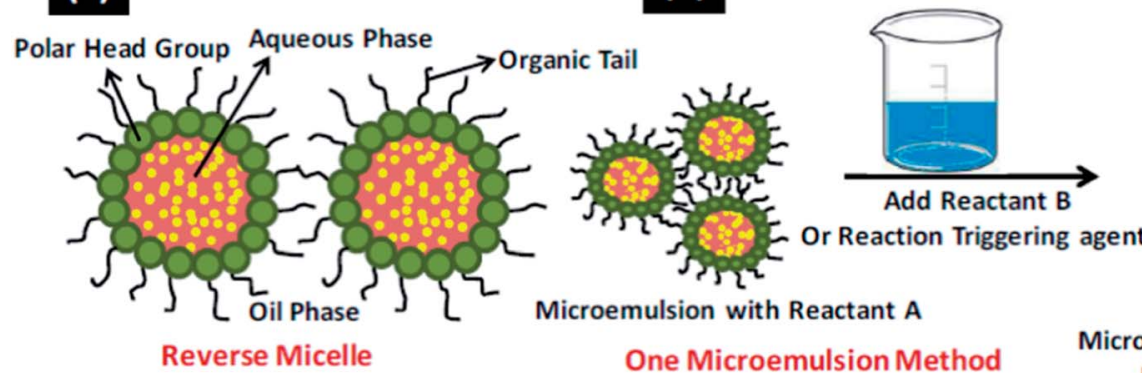

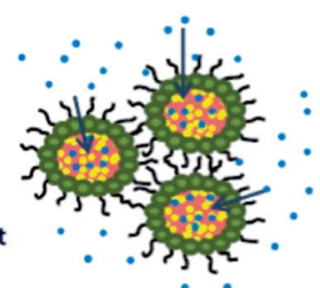

Diffusion of Reactant B across

Microemulsion Interface/Triggering agent Initiates the nucleation process

\section{(c)}

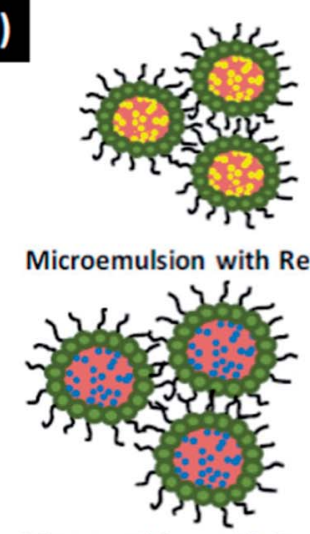

Microemulsion with Reactant B
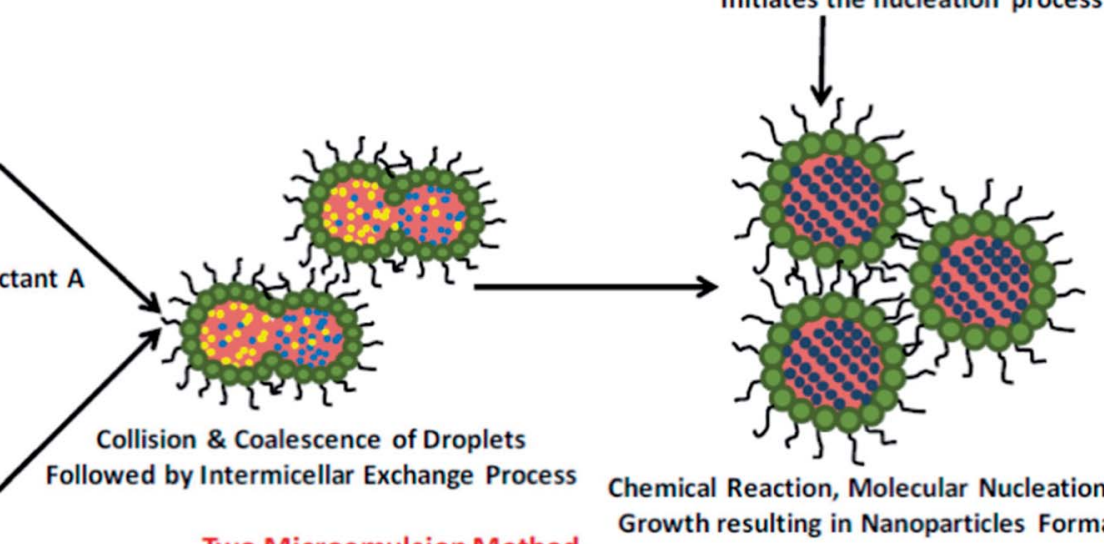

Chemical Reaction, Molecular Nucleation and Growth resulting in Nanoparticles Formation

Scheme $2{ }^{15}$ The general process involved in a revered microemulsion method. (a) A reverse micellar system (b) one microemulsion method and (c) two microemulsion method. Reproduced from ref. 15 with permission from the Royal Society of Chemistry. 
Water splitting is the process of catalytic conversion of liquid water to gaseous oxygen and hydrogen. It is the one of the most widely studied electrocatalytic reactions ${ }^{22}$ and often used as a benchmark to evaluate different electrocatalysts. The overall reaction of water splitting is (eqn (1)):

$$
\mathrm{H}_{2} \mathrm{O}(\mathrm{l}) \Leftrightarrow \mathrm{H}_{2}(\mathrm{~g})+1 / 2 \mathrm{O}_{2}(\mathrm{~g})(\Delta G>0)
$$

Thermodynamically, this reaction is up-hill and nonspontaneous process. The minimum energy required to split one water molecule is $2.46 \mathrm{eV}\left(\Delta G=237 \mathrm{~kJ} \mathrm{~mol}^{-1}\right)$. As overall it is a $2 \mathrm{e}$ process, the minimum potential required to split water is $1.23 \mathrm{~V}$. However, experimentally, most of the electrolysers require a potential greater than this to overcome the electrokinetic barrier of the electrode. Electrochemical water splitting takes place via two half reaction: oxygen evolution reaction (OER) on anode and hydrogen evolution reaction (HER) on cathode (eqn (2) and (3)).

$$
\begin{gathered}
2 \mathrm{H}^{+}+2 \mathrm{e}^{-} \Leftrightarrow \mathrm{H}_{2}(\mathrm{~g}) \text { (water reduction) } \\
2 \mathrm{H}_{2} \mathrm{O}(\mathrm{l}) \Leftrightarrow 4 \mathrm{H}^{+}+4 \mathrm{e}^{-}+\mathrm{O}_{2}(\mathrm{~g}) \text { (water oxidation) }
\end{gathered}
$$

The water oxidation is a $4 \mathrm{e}^{-}$transfer process and thus is kinetically sluggish with a minimum required potential of $1.23 \mathrm{~V}$ (vs. NHE), while HER, a $2 \mathrm{e}$ process is kinetically and thermodynamically facile $(0 \mathrm{~V} v s$. NHE). The overall water splitting is energy driven and this energy may be derived from solar/light energy (photocatalytic process) or electrical energy (electrochemical process) or combination of both solar and electrical energy (photoelectrochemical PEC process). The efficiency of an electrocatalyst for OER or HER may be evaluated on the basis of the many electrocatalytic kinetic parameters such as overpotential $(\eta)$, exchange current density $\left(i_{0}\right)$ and $(c)$ Tafel slope. When an electrochemical reaction proceeds on application of a reasonable potential, it is kinetically controlled. Apart from the required thermodynamic potential, in practice an additional potential to overcome the kinetic factors such as ion mobility (diffusion), circuit resistance and entropy. This excess potential required is termed the overpotential. The intrinsic rate of electron transfer between an analyte in solution and the electrode is reflected by the exchange current density. Thus, the effectiveness of a catalyst for a given electrochemical reaction under a given set of conditions can be quantified. Tafel slopes (b) are derived from the linear portion of the graph of overpotential versus the logarithm of the current density for a particular electrocatalyst catalyzing a particular reaction. Thus, it quantifies the change in electrocatalytic performance of an electrocatalyst over a given potential range. Catalysts with smaller values of the slopes need smaller increments of overpotential to give higher current density, thus making them more effective. While comparing different catalysts, in general, ones with higher $i_{0}$ at lower $\eta$ and a smaller value of $b$ are said to be more active though other factors such as stability under operating conditions and the tendency of the constituent metals to get oxidized at higher $\eta$ has also to be considered. Currently, the benchmark catalyst(s) for HER is Pt/C and for
OER they are $\mathrm{IrO}_{2}$ and $\mathrm{RuO}_{2}$. However, $\mathrm{Pt} / \mathrm{C}$ performs best in acidic medium and has limited OER in alkaline medium. Additionally, $\mathrm{IrO}_{2}$ and $\mathrm{RuO}_{2}$ perform excellent as catalysts for OER in alkaline media in which they have poor HER activity. Research is ongoing to (a) find cheaper alternatives to the currently used precious metal based catalyst for these processes (b) more versatile catalysts e.g. the same catalyst being able to perform multiple transformations like HER and ORR (oxygen reduction reaction), HER and OER and (c) more robust catalysts in terms of stability under acidic or basic conditions.

Our group has produced several classes of inorganic materials, synthesized by hydrothermal or microemulsion routes that demonstrated electrocatalytic water splitting, OER or HER activity. This included (a) metal nanoparticles (b) bimetallic alloy nanoparticles of $\mathrm{Co}, \mathrm{Ni} \mathrm{Fe}$ and $\mathrm{Cu}(\mathrm{c})$ ternary alloy nanoparticles (d) metal cobaltites and manganites as well as (e) metal chalcogenide based composites. The catalysts were adequately characterized and their electrocatalytic activity was evaluated by the usual techniques. In the following sections we shall briefly describe our work and comprehensively review the reports of catalysts of similar nature that have been synthesized by microemulsion and hydrothermal routes and have demonstrated electrocatalytic water splitting, OER and HER activity.

\section{Hydrogen and oxygen evolution (HER and OER) activity of electrocatalysts prepared by hydrothermal and microemulsion methods}

Hydrothermal methods have been used to modify the properties of nanostructures as well as synthesize nanostructures with unique shapes, sizes, morphology and other physical and chemical properties that have shown better electrochemical performance. For e.g. the hydrothermal treatment was used to improve the electrochemical activity of surface oxidized MWCNT for OER, HER and ORR (Fig. 1). ${ }^{23}$ The oxygen content was not appreciably reduced and the significance of this treatment is that this is relatively mild as compared to the high temperature methods. Hydrothermal treatment resulted in enhanced surface hydrophilicity, tuning of the oxygen functional group composition and reduction in defective sites and thus subsequently improvement of electrical conductivity of the MWCNT. This was attributed to increase in $\mathrm{C}=\mathrm{O}$ containing functional groups which have stronger electron withdrawing abilities.

The microemulsion technique, as discussed above is also another widely reported low temperature method used to engineer the morphology, shape, size and such physical characteristics of the nanostructures. These variations in morphology and other physical characteristics have been shown to deeply influence the electrochemical response of these nanostructures. Our group reported the microemulsion synthesis of nanocrystalline $\mathrm{Cu}$ nanoparticles of varying morphology ${ }^{24}$ and evaluated their HER and OER performance. 

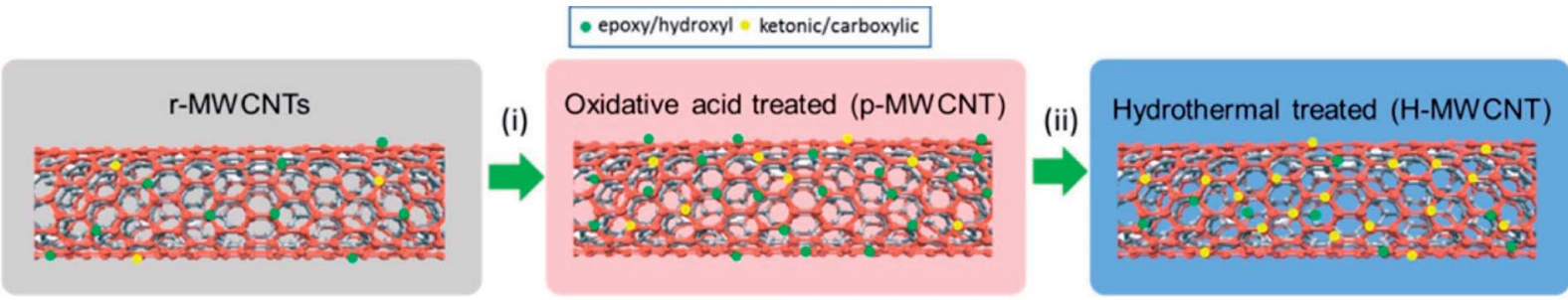

(i) Acid oxidation in a Piranha mixture $\left(75 \mathrm{ml} \mathrm{H}_{2} \mathrm{SO}_{4}, 25 \mathrm{ml} \mathrm{30 \%} \mathrm{H}_{2} \mathrm{O}_{2}\right), 5$ hours, $25^{\circ} \mathrm{C}$.

(i) Hydrothermal treatment at various temperature for 18 hours.
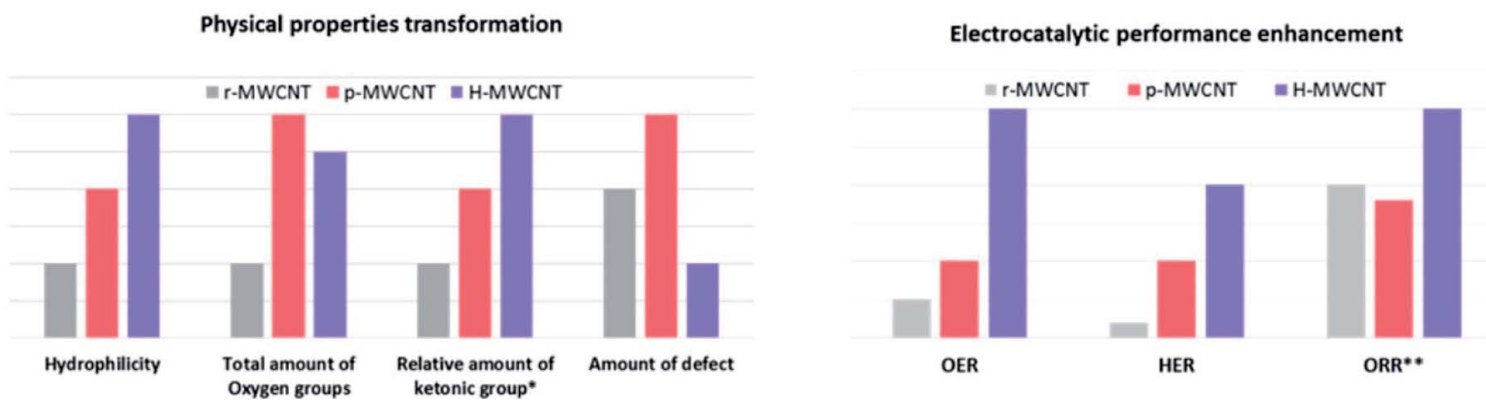

Fig. 1 Enhancement of electrocatalytic property and modification of physical properties by hydrothermal treatment. ${ }^{23}$ Reprinted with permission from ACS Appl. Mater. Interfaces, 2016, 8(51), 35513-35522. Copyright 2016 American Chemical Society.

Nanocubes ( $\sim 50 \mathrm{~nm}$ ) nanorods (diameter of $\sim 3 \mathrm{~nm}$ and length of $\sim 50 \mathrm{~nm})$ and nanospheres $(5 \mathrm{~nm})$ were synthesized and subsequently reduced at $400{ }^{\circ} \mathrm{C}$ in a hydrogen atmosphere. It was observed that the cube-shaped nanoparticles show significantly higher hydrogen and oxygen evolution efficiencies as compared to the nanorods and spherical nanoparticles. The nanospheres show better hydrogen and oxygen evolution efficiencies than the nanorods, though from the similar Tafel slopes $(-0.16 \mathrm{mV}$ per decade to $-0.25 \mathrm{mV}$ per decade) it was inferred that the mechanism of electron transfer is same for all morphologies. HER reactions were carried out in $0.1 \mathrm{M} \mathrm{Cl}^{-}$ while OER were carried out in $0.1 \mathrm{M} \mathrm{KOH}$. We also reported the synthesis of $\mathrm{Cu}$ nanoparticles from $\mathrm{Cu}$-oxalate precursors synthesized by microemulsion techniques. ${ }^{25}$ HER and OER experiments were carried out in alkaline medium. In the case of HER the current densities were found to be $12 \mathrm{~mA} \mathrm{~cm}^{-2}$ (over glassy carbon electrode) and $46 \mathrm{~mA} \mathrm{~cm}{ }^{-2}$ (over Pt electrode) which were significantly higher than previously reported values (maximum $1 \mathrm{~mA} \mathrm{~cm}^{-2}$ ). For OER, the current density was found to be $1.6 \mathrm{~mA} \mathrm{~cm}^{-2}$ (that was slightly higher) for the glassy carbon electrode and $15 \mathrm{~mA} \mathrm{~cm}{ }^{-2}$ for $\mathrm{Pt}$ as the working electrode, values that were 4-30 times higher than earlier reports. The high efficiency was thought to be related to the high surface area $\left(34 \mathrm{~m}^{2} \mathrm{~g}^{-1}\right)$ of these crystalline $\mathrm{Cu}$ nanoparticles. Additionally, these particles show good stability as electrocatalysts and retain their activity after even 50 cycles.

\subsection{Binary alloys}

Our group reported the synthesis of $\mathrm{Cu}-\mathrm{Co}$ core-shell nanoparticles $^{26}$ by microemulsion methods and evaluated the electrochemical activity of these particles by means of HER by splitting water. Electrochemical studies of these nanocrystalline $\mathrm{Cu}-\mathrm{Co}$ alloy particles indicate higher HER efficiencies $(\sim 5$ times) compared to bulk (micrometer-sized) $\mathrm{Cu}-\mathrm{Co}$ alloy particles. We also reported that binary Fe-Co nanoparticles show significant enhancement of electrocatalytic activity as compared to bulk alloys. ${ }^{27}$ The microemulsion based synthesis of these alloys was reported for the first time and various alloys of the composition $\mathrm{Fe}_{75} \mathrm{Co}_{25}, \mathrm{Fe}_{67} \mathrm{Co}_{33}, \mathrm{Fe}_{50} \mathrm{Co}_{50}$, and $\mathrm{Fe}_{33} \mathrm{Co}_{67}$ with an average size of $20,25,10$, and $40 \mathrm{~nm}$, respectively, were obtained. Nanoparticles of the alloy with composition $\mathrm{Fe}_{33} \mathrm{Co}_{67}$ exhibit the highest hydrogen and oxygen evolution efficiencies ( $\sim 100$ times higher) as compared to other $\mathrm{Fe}-\mathrm{Co}$ alloys in nanocrystalline or in bulk form (Fig. 2). It was postulated that this composition performs best due to the higher concentration of Co, which was reported to be a better electrocatalyst. NiTe nanostructures anchored on three-dimensional nickel foam were synthesized using the microemulsion method were reported as high-performance catalyst for electrochemical water oxidation by Li $e t a l .^{28}$ The high efficiency was attributed to the 2D NiTe ultrathin nanosheets which have large number of exposed active sites and 3D hierarchical porous structure.

\subsection{Ternary alloys}

Our group has reported the HER activity of two ternary alloy catalysts ${ }^{17,29}$ both prepared by the microemulsion method. $\mathrm{Cu}-\mathrm{Fe}-$ $\mathrm{Ni}$ ternary alloy ${ }^{29}$ nanoparticles of varying compositions $(1: 1: 1$, $1: 2: 1$ and $1: 1: 2)$ and sizes $(55-80 \mathrm{~nm})$ were prepared using CTAB as the surfactant, 1-butanol as co surfactant and iso-octane as the oil phase. When these nanostructured alloys were evaluated 


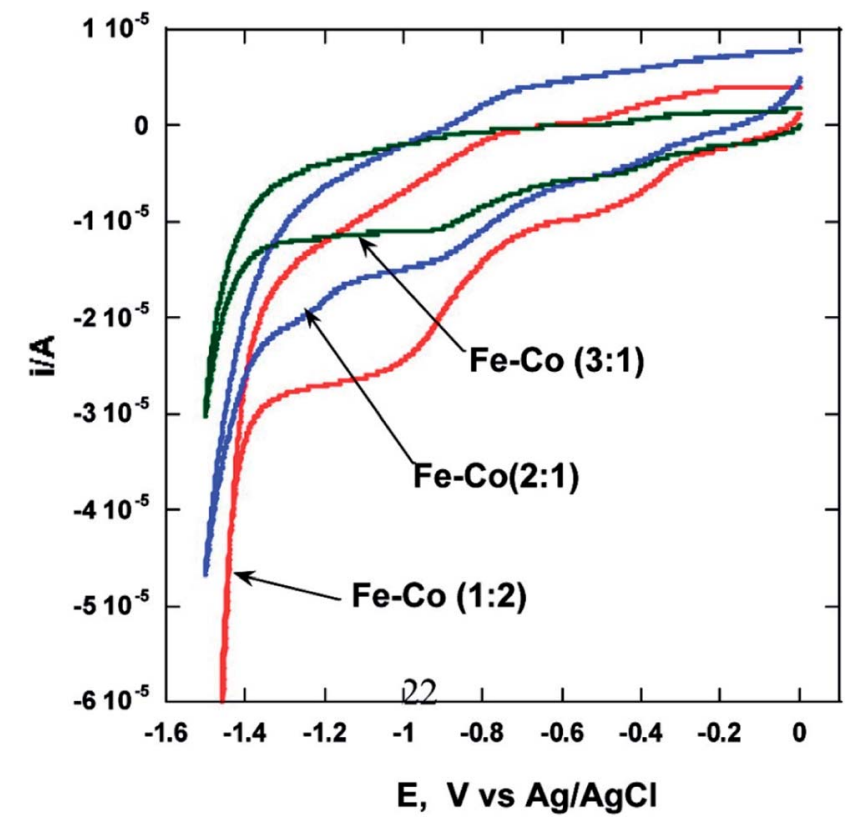

Fig. 2 Cyclic voltammograms showing the effect ${ }^{27}$ of composition on the electrocatalytic properties of $\mathrm{Fe}-\mathrm{Co}$ alloys. Reprinted with permission from J. Phys. Chem. C, 2010, 114(44), 18779-18784. Copyright 2010 American Chemical Society.

for HER in a basic medium a very high current density of $228 \mathrm{~mA}$ $\mathrm{cm}^{-2}$ at an overpotential of $545 \mathrm{mV}$ with an onset of $-267 \mathrm{mV}$ was achieved for the $1: 1: 1$ alloy. Two metals, one with weak $\mathrm{M}-\mathrm{H}$ binding affinity (Fe) and the other with strong $\mathrm{M}-\mathrm{H}$ binding affinity $(\mathrm{Cu})$ were alloyed with $\mathrm{Ni}$, which is the most appropriate choice for a low cost catalyst for HER. Spherical $\mathrm{Cu}-\mathrm{Co}-\mathrm{Ni}$ alloy nanoparticles ${ }^{17}$ of composition, $1: 1: 1,1: 1: 2$ and $1: 2: 1$ were synthesized by reverse micellar method. These were evaluated for HER activity in the acidic medium with the $1: 1: 1$ composition demonstrating the best performance exhibiting a low onset overpotential $(257 \mathrm{mV})$ and a small Tafel slope $\left(102 \mathrm{mV} \mathrm{dec}^{-1}\right)$ at current densities of $-10 \mathrm{~mA} \mathrm{~cm}{ }^{-2}$. The catalyst was stable for over 500 cycles. Xie et al. ${ }^{30}$ performed hydrothermal sulfurization on the surface of AgCuZn alloy to obtain AgCuZn sulfide nanoparticles, which on electrochemical treatment resulted in $\mathrm{CuO}-\mathrm{Ag}_{2} \mathrm{O}$ nanowire arrays on the alloy surface. The composites were reported to be efficient and stable electrocatalysts for water splitting OER reactions. An Au based alloy, AuCuCo prepared hydrothermally ${ }^{31}$ was reported to exhibit comparable electrocatalytic activity and better stability towards OER in alkaline media as compared to commercial Pt/C and $\mathrm{IrO}_{2} / \mathrm{C}$ catalyst. Additionally, this catalyst was active for ORR too. The improved electrocatalytic activity of AuCuCo was attributed to its unique three-dimensional structure, the abundance of twin crystals, and change in the electronic structure of $\mathrm{Au}$ by alloying. However, the exact reasons for better performance at this juncture requires further studies for a complete understanding.

\subsection{Metal chalcogenides and supported metal chalcogenides}

The most studied material for electrochemical applications that falls in this category is $\mathrm{MoS}_{2}$. Our group reported $\mathrm{MoS}_{2}$ functionalized $\mathrm{Mo}_{2} \mathrm{~N}$ nanostructures. ${ }^{32}$ The $\mathrm{MoS}_{2}$ nanosheets were prepared by hydrothermal methods. Additionally, different morphologies of $\mathrm{Mo}_{2} \mathrm{~N}$ (hexagonal, triangular and nanowires) were also synthesized by hydrothermal methods. HER activity of the composites $\left(\mathrm{MoS}_{2}-\mathrm{Mo}_{2} \mathrm{~N}\right)$ were evaluated in an acidic medium and it was determined that the presence of interfaces and junctions contributed significantly to the electrocatalytic activity as evident from the low charge transfer resistance. It was also demonstrated that the HER activity of the $\mathrm{Mo}_{2} \mathrm{~N}-\mathrm{MoS}_{2}$ composites is superior as compared to the bare $\mathrm{MoS}_{2}$ and $\mathrm{Mo}_{2} \mathrm{~N}$ nanostructures. Additionally the triangular composites had the best electrochemical response (Fig. 3).

A Co doped $2 \mathrm{H}-\mathrm{MoS}_{2}$ ultrathin nanomesh with high defect concentration and large porosity was demonstrated to improve the electrochemical performance for HER. ${ }^{33} \mathrm{~A}$ 3D reduced graphene oxide (RGO) scaffold acted as channels for fast electron and ion transport. This 3D defect-rich $\mathrm{MoS}_{2}$ nanomesh/RGO foam can be easily obtained through a one-pot cobalt acetate/ graphene oxide (GO) co-assisted hydrothermal reaction, in which GO, Co and acetate ions are co-morphology-controlling agents and defect inducers. The experimental studies were complemented by DFT calculations that indicate that the defects result in formation of $\mathrm{Co}-\mathrm{Mo}-\mathrm{S}$ structures, thus releasing inert basal planes to active sites. Layer expanded $\mathrm{MoS}_{2}$ nanosheets/carbon fiber nanocomposites were synthesized by the hydrothermal method by an in situ reduction of ammonium tetrathiomolybdate on carbon fibers and evaluated for HER. ${ }^{34}$ The HER studies show that the composites with Mo/C molar ratio of 0.04 has the highest catalytic activity with the lowest overpotential (131 mV vs. RHE) and the highest current density $\left(10 \mathrm{~mA} \mathrm{~cm} \mathrm{~cm}^{-2}\right.$ at $200 \mathrm{mV}$ vs. RHE). This was attributed to a balance between the number of exposed active edge sites of $\mathrm{MoS}_{2}$ and fast transport paths for electrons provided by carbon fibers. EIS spectra indicate low charge transfer resistance indicating fast faradaic process and superior HER studies. Composites of graphene with amorphous transition-metal chalcogenides $\left(\mathrm{MoS}_{x}, \mathrm{WS}_{x}\right)$ that were synthesized by hydrothermal techniques were reported as electrocatalysts for HER in acidic media. ${ }^{35} \mathrm{MoS}_{x} /$ graphene composites perform much superior to $\mathrm{WS}_{x}$ /graphene composites. This was attributed to the surface oxidation in the $\mathrm{WS}_{x}$ based composites, as inferred from XPS studies. The onset potential was close to the HER onset potential of industrially used platinum catalysts. $\mathrm{MoS}_{2} /$ $\mathrm{Ni}_{3} \mathrm{~S}_{2}$ nanorod arrays aligned on $\mathrm{Ni}$ foam (Fig. 4) were demonstrated to function as electrodes for overall water splitting reaction. ${ }^{36}$ The catalysts were stable and a high current density at low overpotential was achieved. To achieve current density of $10,100,200$ and $300 \mathrm{~mA} \mathrm{~cm}{ }^{-2}$ in an electrolyzer, potentials of $1.467,1.593,1.640$ and 1.661 are required. EIS studies indicate low charge transfer resistance indicating fast electron transport efficiency in the composite.

Li et al. ${ }^{37}$ reported $\mathrm{SnS}_{2}$ modified $\mathrm{TiO}_{2}$ nanobelt based electrocatalysts which displayed significant enhancement of electrocatalytic properties w.r.t. OER. This performance was explained based on large contact area of the $\mathrm{TiO}_{2}$ nanobelts and the stability of the $\mathrm{SnS}_{2}$ nanosheets. Additionally, the exposed crystal facets of $\mathrm{TiO}_{2}$ and the change in "valence bond 

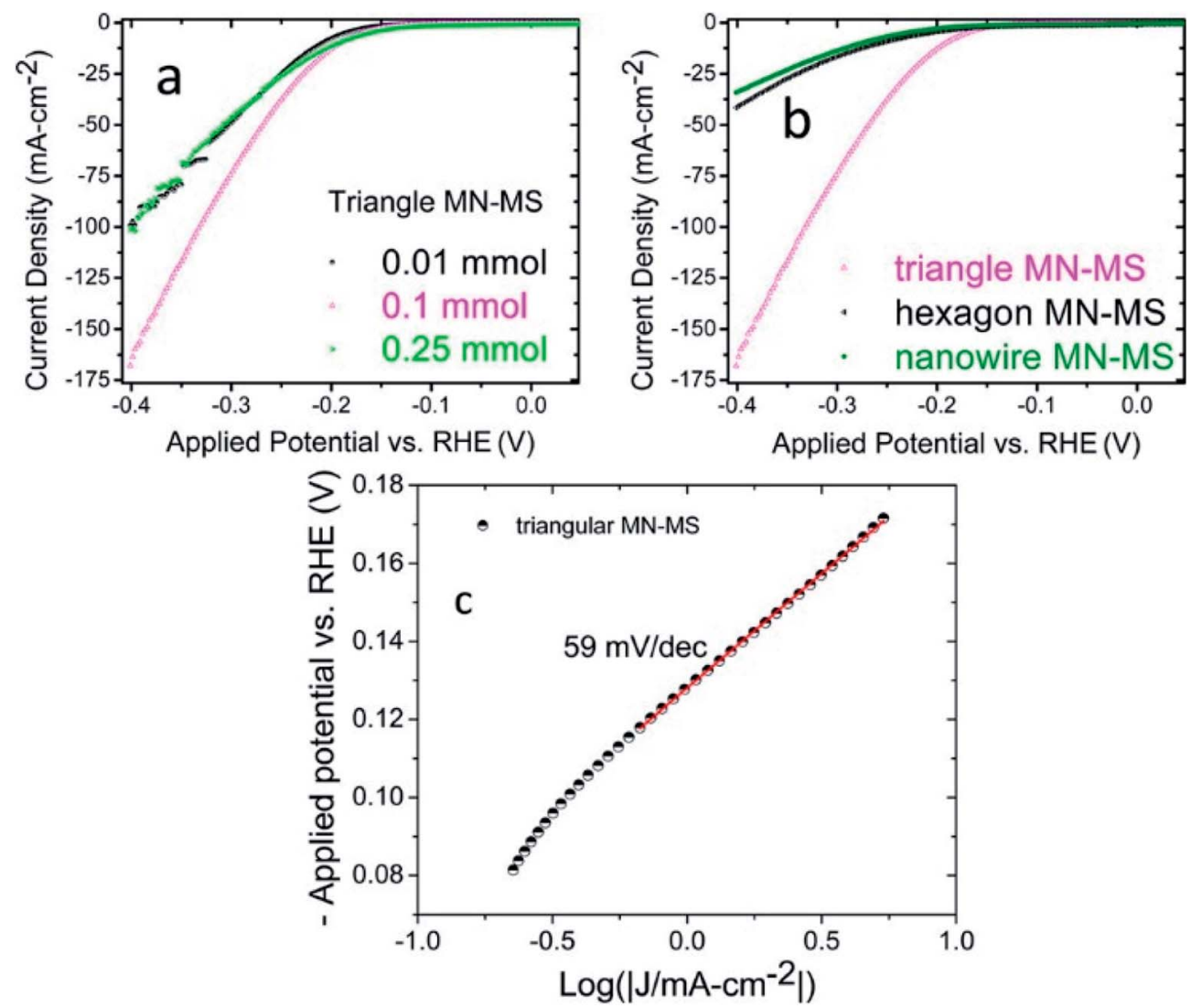

Fig. 3 (a) Linear sweep voltammetry graphs depicting the current densities of triangular MN-MS composites with various components of MoS 2 (b) LSV curves of $\mathrm{Mo}_{2} \mathrm{~N}-\mathrm{MoS}_{2}$ components with differing morphologies (c) Tafel plot obtained from the LSV curve of triangular MN-MS. A Tafel slope of $59 \mathrm{mV}$ per decade is observed. ${ }^{32}$ Reprinted with permission from ACS Appl. Mater. Interfaces, 2017, 9(23), 19455-19461. Copyright 2017 American Chemical Society.

environment" due to formation of heterostructures was attributed to the electrochemical properties. An overpotential of $570 \mathrm{mV}$ with a current density of $10 \mathrm{~mA} \mathrm{~cm}{ }^{-2}$ and a low Tafel slope of $107 \mathrm{mV}$ per decade was reported. Nickel sulfide nanosheets directly grown on $\mathrm{Ni}$ foam were reported to be efficient and stable as electrocatalysts used for water reduction and oxidation in alkaline medium. ${ }^{38}$ A hydrothermal-sulfurization method in $\mathrm{Na}_{2} \mathrm{~S}$ solution was used to obtain $\mathrm{Ni}_{3} \mathrm{~S}_{4}$ nanosheets directly grown on Ni foam. For HER, only a low overpotential of $-122 \mathrm{mV}$ is needed to achieve a current density of $10 \mathrm{~mA} \mathrm{~cm}{ }^{-2}$ with a small Tafel slope of $69 \mathrm{mV} \mathrm{dec}{ }^{-1}$. For OER, it delivers a current density of $20 \mathrm{~mA} \mathrm{~cm}{ }^{-2}$ at the overpotential of $320 \mathrm{mV}$ and a Tafel slope of $71 \mathrm{mV} \mathrm{dec}^{-1}$. When used in an electrolyzer (catalyst acts as both an anode and cathode), for overall water splitting, a potential of $1.61 \mathrm{~V}$ was required to achieve a current density of $10 \mathrm{~mA} \mathrm{~cm}{ }^{-2}$; while the corresponding potential required to achieve a current density of $20 \mathrm{~mA} \mathrm{~cm}^{-2}$ was $1.67 \mathrm{~V}$. The high electrocatalytic activity was attributed to the facile mass transport due to the unique 3D structure. $\mathrm{Ni}_{3} \mathrm{~S}_{2} /$ ballmilled silicon powder was prepared by hydrothermal techniques and was reported as a bi-functional electrocatalyst for HER and OER. ${ }^{39}$ The catalyst was prepared by grafting heazlewoodite $\left(\mathrm{Ni}_{3} \mathrm{~S}_{2}\right)$ on the silicon powder $\left(\left(\mathrm{Ni}_{3} \mathrm{~S}_{2} / \mathrm{Si}\right)\right.$ - ball-milled and a control non-ball milled) silicon powder through a hydrothermal process. The products were then etched using the HF solution to prepare the modified porous silicon based composites. These catalysts exhibited electrocatalytic activity towards both alkaline and acidic HER with Tafel slopes of 74 and $52 \mathrm{mV} \mathrm{dec}^{-1}$ respectively. An electrocatalytic activity for the OER (in $1 \mathrm{M} \mathrm{KOH}$ ) with an overpotential of $164 \mathrm{mV} \mathrm{dec}^{-1}$ was reported. The catalyst with a porous structure and a ball milled support exhibited the highest efficiency. The use of this bifunctional catalyst in an overall water splitting electrolyzer was not discussed.

As mentioned earlier, most of the studies conducted usually deal with synthesis of new nanostructures and/or composites and evaluation of their activity towards OER or HER reaction. Thereafter, based on the activity, qualitative explanations based on interaction of the components of the composite or those based on surface area are used to explain the activity of the materials. It is essential to have a more focused study to understand the efficiency of a specific nanocomposite towards HER and OER reactions. Chia et al. ${ }^{40}$ performed a detailed study on the role of the structural features of layered orthorhombic $\mathrm{SnS}$ as compared to hexagonal $\mathrm{SnS}_{2}$ that led to higher inherent electro-activity in $\mathrm{SnS}_{2}$. Three distinct cathodic signals were obtained in $\mathrm{SnS}_{2}$ as opposed to a broad reduction peak in the largely electro-inactive SnS. Additionally, $\mathrm{SnS}_{2}$ exhibits a faster 


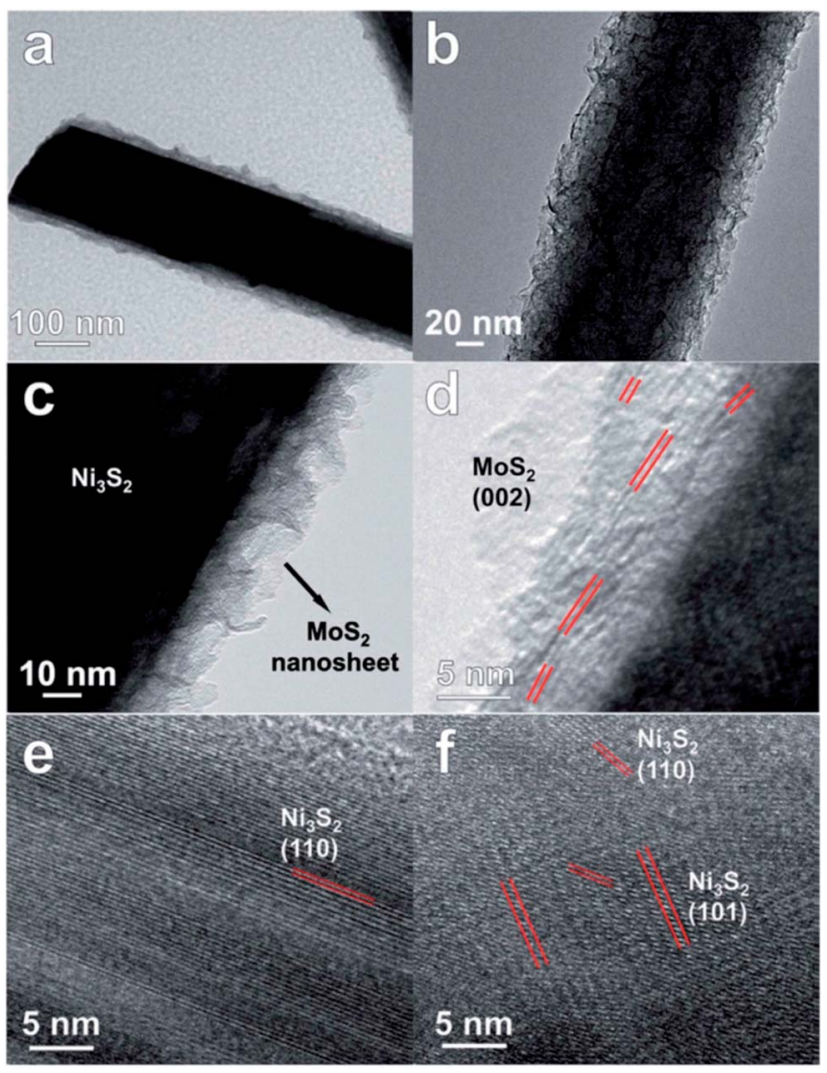

Fig. 4 (a-c) TEM and (d-f) HRTEM images of $\mathrm{MoS}_{2} / \mathrm{Ni}_{3} \mathrm{~S}_{2}$ nanorod arrays aligned on $\mathrm{Ni}$ foam. Reproduced from ref. 36 with permission from the Royal Society of Chemistry.

heterogeneous electron transfer (HET) rate than SnS. It may be mentioned though that both $\mathrm{SnS}$ and $\mathrm{SnS}_{2}$ show much lower current density than the glassy carbon electrode. For the OER and ORR, these electrocatalysts do not perform well. However, both these catalysts perform very well as HER electrocatalysts, their performance surpassing the GC electrode. Amongst the two chalcogenides, $\mathrm{SnS}_{2}$ has better performance for the HER. The difference in HER performance was investigated and explained by means of HER electrode kinetics and density functional theory (DFT) calculation. By means of electrochemical impedance spectroscopy (EIS), $\mathrm{SnS}_{2}$ demonstrated a significantly faster HER kinetics than SnS. The DFT study unveiled that the high electrocatalytic performance of $\mathrm{SnS}_{2}$ originated from the favorable $\Delta G_{\mathrm{H}}$ at the S edges. $\Delta G_{\mathrm{H}}$ of SnS at all edges are disadvantageous for HER. In another study, ${ }^{41}$ the absolute electronic energy levels have been investigated in $\mathrm{AgInS}_{2}$ by varying the relative composition of the elements and crystallographic phases. These structures were synthesized using solvothermal methods and found to have a good electrocatalytic activity towards HER that were strongly dependent on the composition and the crystal structure of these nanocrystals. The monoclinic structures were found to be the most active as HER electrocatalyst, whereas cubic $\mathrm{AgInS}_{2}$ was observed to be the least active of the studied crystallographic phases and compositions. The electrocatalytic activity of the monoclinic structures is almost comparable to the $\mathrm{MoS}_{2}$-based nanostructures reported in the literature. From these studies it was understood that the Fermi level of these nanocrystals is an important factor for deciding the electrocatalytic efficiency. $\mathrm{Ni}$ Fe sulfides supported on Ni foam (NF) were synthesized by a hydrothermal sulfurization method and reported as efficient catalysts for OER. ${ }^{42} \mathrm{NiFeS} / \mathrm{NF}$ exhibits an overpotential of $65 \mathrm{mV}$ at $10 \mathrm{~mA} \mathrm{~cm}^{-2}$, much lower than most Ni-based electrocatalysts. Additionally, at $100 \mathrm{~mA} \mathrm{~cm}{ }^{-2}$ the overpotential is $189 \mathrm{mV}$ suggesting promising OER activity for large scale applications. However, the catalyst suffers from stability issues and to remedy the situation a layer of Fe was electrodeposited on this, which however resulted in lower performance.

Monoclinic $\mathrm{Co}_{3} \mathrm{Se}_{4}$ nanowire electrodes on cobalt foam $\left(\mathrm{Co}_{3} \mathrm{Se}_{4} / \mathrm{CF}\right)$ were synthesized by a hydrothermal method using selenourea and evaluated for OER. ${ }^{43}$ In an electrolyzer where $\mathrm{Co}_{3} \mathrm{Se}_{4} / \mathrm{CF}$ acted both as a cathode and anode respectively for overall water splitting, a potential of $1.55 \mathrm{~V}$ was required for a current density of $10 \mathrm{~mA} \mathrm{~cm}{ }^{-2}$ while for a current density of 20 $\mathrm{mA} \mathrm{cm} \mathrm{cm}^{-2}$ a potential of $1.63 \mathrm{~V}$ was required. Significantly, the electrolyzer operated at $10 \mathrm{~mA} \mathrm{~cm}^{-2}$ for over $3500 \mathrm{~h}$ and at 100 $\mathrm{mA} \mathrm{cm}{ }^{-2}$ for at least $2000 \mathrm{~h}$ without noticeable degradation.

\subsection{Metal oxides}

Our group has reported the electrocatalytic properties of nanostructured dimagnesium manganese oxide $\left(\mathrm{Mg}_{2} \mathrm{MnO}_{4}-\right.$ spinel structure) obtained from metal oxalate precursors synthesized by a microemulsion route. ${ }^{44}$ The anisotropy of the metal oxalate is retained in the oxide and rod shaped structures are obtained. These nanostructured $\mathrm{Mg}_{2} \mathrm{MnO}_{4}(\mathrm{Mn}$ is in +IV state) are efficient anode materials for OER displaying a current density of $14 \mathrm{~mA} \mathrm{~cm}{ }^{-2}\left(v s . \mathrm{Ag} / \mathrm{Ag}^{+}\right)$. The anodes are stable over at least 30 cycles of oxidation and reduction in an alkaline medium. Cobalt magnetites $\left(\mathrm{Mn}_{x} \mathrm{Co}_{3-x} \mathrm{O}_{4}, x=1\right)$ based electrocatalysts was reported earlier by our group for water splitting applications. ${ }^{45}$ These magnetites were synthesized by hydrothermal methods and two nanocrystalline phases, cubic $\mathrm{Co}_{2} \mathrm{MnO}_{4}$ and tetragonal $\mathrm{CoMn}_{2} \mathrm{O}_{4}$ were successfully synthesized by varying the stoichiometric ratio of Co and Mn salts. The cubic $\mathrm{Co}_{2} \mathrm{MnO}_{4}$ nanocubes displayed five times higher activity towards ORR than tetragonal $\mathrm{CoMn}_{2} \mathrm{O}_{4}$ nanocubes while the latter phase is a better electrocatalyst towards OER (Fig. 5). XPS analysis shows the presence of two types of $\mathrm{O}$ on the surface, (lattice $\mathrm{O}$ and surface adsorbed $\mathrm{O}$ ) and the electrocatalytic activity can be correlated to the oxygen binding ability on the catalyst surface. Additionally, it was determined that Co(III) and Mn(III) dominated over Mn(Iv).

Orthorhombic molybdenum oxide $\left(\alpha-\mathrm{MoO}_{3}\right)$ nanobelts that were greater than $10 \mathrm{~nm}$ wide and less than $200 \mathrm{~nm}$ long were synthesized by hydrothermal methods. ${ }^{\mathbf{4 6}}$ These belts displayed satisfactory properties for HER reactions and their electrochemical performance (evaluated by means of Tafel plots) was $\sim 1.7$ times superior to the commercially available $\mathrm{MoO}_{3}$. Co or $\mathrm{Ru}$ doped $\alpha-\mathrm{MnO}_{2}$ nanorods were produced by a continuous hydrothermal synthesis process $\left(10 \mathrm{~g} \mathrm{~h}^{-1}\right)$ and were reported to be efficient catalysts for OER reactions. ${ }^{47}$ DFT calculations complemented experimental studies to evaluate the role of the 


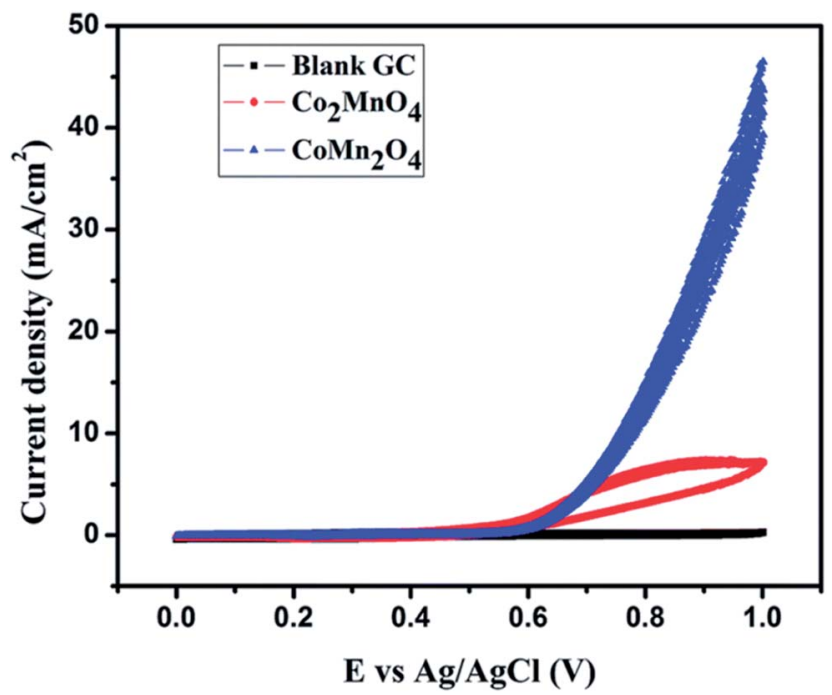

Fig. $5{ }^{45} \mathrm{Cyclic}$ voltammogram depicting OER activity of $\mathrm{CO}_{2} \mathrm{MnO}_{4}$ in $1 \mathrm{M} \mathrm{KOH}$ electrolyte. Reproduced from ref. 45 with permission from the Royal Society of Chemistry.

dopants on $\alpha-\mathrm{MnO}_{2}$ support. The OER and ORR activity were found to be comparable to the commercially available $\mathrm{RuO}_{2}$ and $\mathrm{Pt} / \mathrm{C}$ catalysts. Ultrafine $\mathrm{PtO}_{2}$ nanoparticles coupled with a $\mathrm{Co}(\mathrm{OH}) \mathrm{F}$ nanowire array on a $\mathrm{TiO}_{2}$ mesh were prepared by hydrothermal techniques and were evaluated for electrochemical HER. ${ }^{48}$ This composite was reported to be highly active and durable for the HER in alkaline media (Fig. 6). This high efficiency has been attributed to a strong synergetic effect between spatially separated ultrafine $\mathrm{PtO}_{2}$ nanoparticles and $\mathrm{Co}(\mathrm{OH}) \mathrm{F}$. The above hydrothermal process, wherein anion exchange of $\left[\mathrm{PtCl}_{6}\right]^{2-}$ with $\mathrm{OH}^{-}$leads to an in situ generation of $\mathrm{PtO}_{2}$ nanoparticles on $\mathrm{Co}(\mathrm{OH}) \mathrm{F}$ nanoarray/titania mesh, is simple and maybe easy to be scaled up. DFT calculations indicated a nearly barrierless water dissociation and optimal free energy of $\mathrm{H}$ adsorption $\left(\Delta G_{\mathrm{H}^{*}}\right)$ on the interface of $\mathrm{PtO}_{2}$ and $\mathrm{Co}(\mathrm{OH}) \mathrm{F}$. However further investigation on the nature and identity of the active site is warranted to obtain an in-depth insight of the reaction mechanism. Chen et $a{ }^{49}$ studied the OER activity of $\mathrm{Co}_{3} \mathrm{O}_{4}$ nanoflakes synthesized using microwave assisted hydrothermal methods (Scheme 3). These mesoporous nanoflakes exhibited OER active properties as well as good catalytic stability. This was attributed to both the stability of unique mesoporous structure and highly reactive facets. The active electrocatalyst was postulated to be a $\mathrm{Co}(\mathrm{Iv})$ species.

Hydrothermal synthesis of $\mathrm{Co}_{3} \mathrm{O}_{4}$ nanowire electrocatalysts for oxygen evolution reaction was reported. ${ }^{50}$ Glycerol was used as a morphology directing agent which led to short nanorods in $\sim 4 \mathrm{~h}$ while a longer reaction $(\sim 12-16 \mathrm{~h})$ produced longer rods which were termed as nanowires. The aspect ratio of the nanowires after $16 \mathrm{~h}$ reaction time was $28: 1$. After $\sim 24 \mathrm{~h}$ it was observed that the nanowires had assembled into bundles. The $\mathrm{Co}_{3} \mathrm{O}_{4}$ nanowires obtained after $\sim 12 \mathrm{~h}$ of reaction were found to be electrocatalytically most active due to highest surface area. A close correlation of the OER catalytic activity is observed with the oxidation state and the density of active sites of the catalyst exposed to the electrolyte solution. Though both $\mathrm{Co}_{3} \mathrm{O}_{4}$ nanowires and nanoparticles exhibited a considerable loss in OER
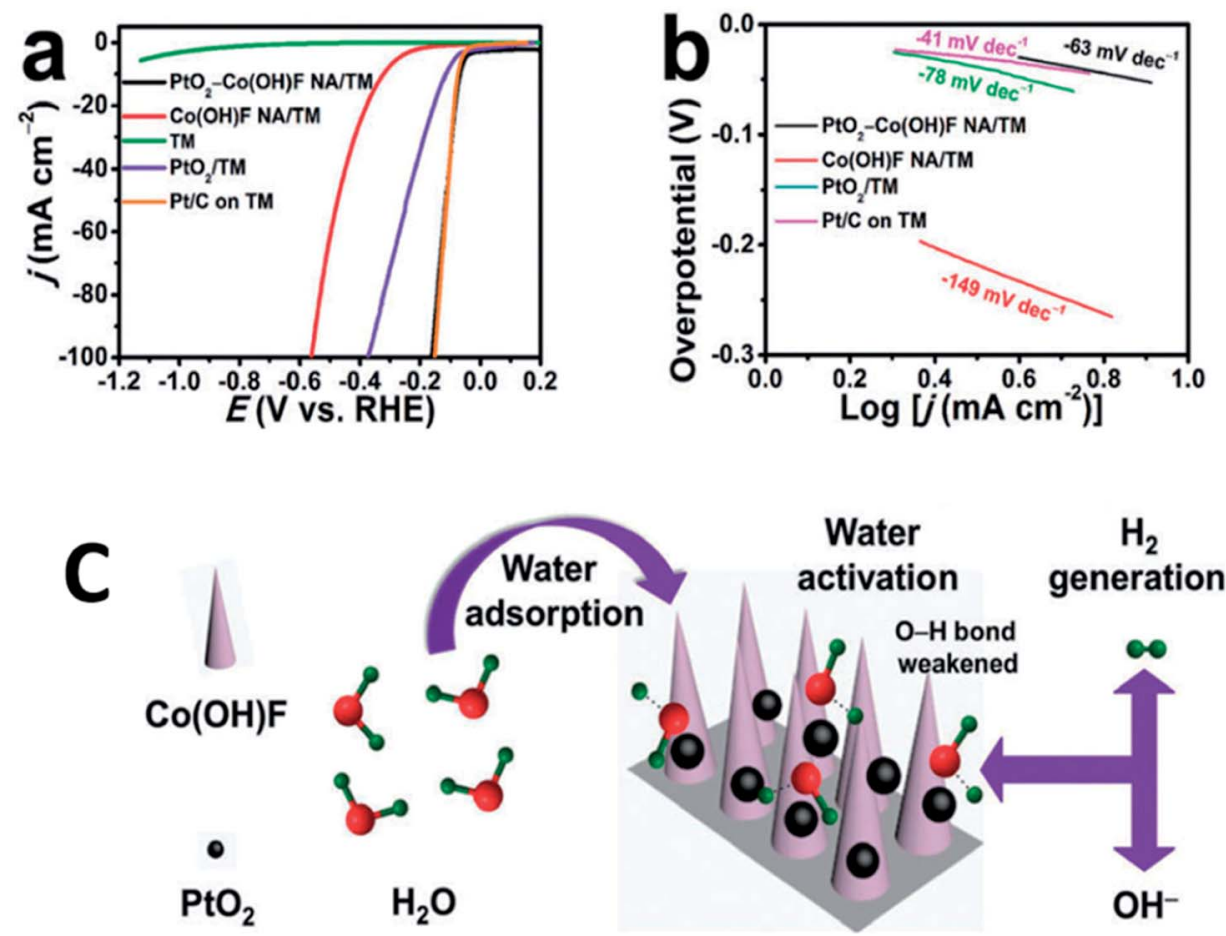

Fig. 6 (a) LSV curves recorded at a scan rate of $5 \mathrm{mV} \mathrm{s}^{-1}$ and (b) the resulting Tafel plots. (c) Schematic of water adsorption, water activation, and hydrogen generation processes for $\mathrm{PtO}_{2}-\mathrm{Co}(\mathrm{OH}) \mathrm{F} \mathrm{NA} / \mathrm{TM}$. Nano Array = NA; Titania Mesh = TM. Reproduced from ref. 48 with permission from the Royal Society of Chemistry. 


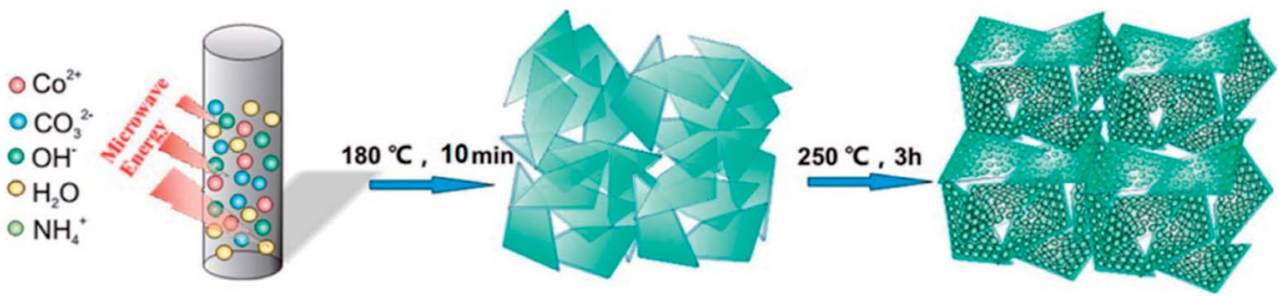

Microwave assisted

hydrothermal synthesis

$\mathrm{Co}\left(\mathrm{CO}_{3}\right)_{0.5}\left(\mathrm{OH}_{0.11} \mathrm{H}_{2} \mathrm{O}\right.$

Mesoporus $\mathrm{CO}_{3} \mathrm{O}_{4}$

Scheme 3 Synthesis of mesoporous $\mathrm{CO}_{3} \mathrm{O}_{4}$ nanoflakes ${ }^{49}$ using microwave-assisted hydrothermal technique. Reprinted with permission from ACS Appl Mater Interfaces 2015, 7(5), 3306-13. Copyright 2015 American Chemical Society.

activity during the stability studies, the former produced higher catalytic current than the latter which was attributed to a slightly higher stability. NiO nanoparticles supported on C nanobelts were synthesized by direct oxidation of $\mathrm{Ni}$ foam using hydrothermal techniques and these composites were found to be active for both OER and HER. ${ }^{51}$ The NiO nanobelt/C has a much lower HER efficiency than the bare NiO nanobelt electrodes and Pt electrodes which was attributed to a layer of nanocarbon deposition on the nanobelt. However, the OER performance was better for the composite due to the $\mathrm{N}$ doped $\mathrm{C}$ layer. The source of $\mathrm{N}$ was dopamine that was used as a precursor for the $\mathrm{C}$ supports. The $\mathrm{C}$ layer was also predicted to mechanically protect the nanostructure and enhance electrochemical stability. However, an indepth study is required to elucidate the role of the $\mathrm{C}$ and $\mathrm{N}$ on the electrocatalytic process. Even though the same catalyst was active for both HER and OER, in this report its performance for overall water splitting activity in an electrolyzer (where the same catalyst is used as a cathode and anode in a single cell) was not evaluated. $\mathrm{NiCo}_{2} \mathrm{O}_{4}$ rods over $\mathrm{Co}_{3} \mathrm{O}_{4}$ nanosheets synthesized by a two-step hydrothermal method were reported as efficient catalyst for water splitting reactions. ${ }^{52}$ The OER activity of the composite (peak current: $4.18 \mathrm{~mA} \mathrm{~cm}{ }^{-2}$ ) was an approx. 2.25 times higher than that of commercial $\mathrm{RuO}_{2}$ catalyst $\left(1.84 \mathrm{~mA} \mathrm{~cm}{ }^{-2}\right)$. The satisfactory electrochemical performance of the composite material was attributed to the oxygen-deficient $3 \mathrm{D}$ architecture providing more catalytic active sites. $\mathrm{NiCO}_{2} \mathrm{O}_{4}$ nanowire arrays ${ }^{53}$ prepared by hydrothermal techniques were reported to catalyze the OER in $1 \mathrm{M} \mathrm{KOH}$ electrolyte. The catalyst exhibits higher catalytic activity than $\mathrm{Co}_{3} \mathrm{O}_{4}$ and $\mathrm{NiO}$ which is attributed to a larger surface area, one-dimensional architecture, higher conductivity and synergy between the components in the bifunctional catalyst. The current density observed was $90 \mathrm{~mA} \mathrm{~cm}{ }^{-2}$ at $0.9 \mathrm{~V} v s$. SCE and the Tafel slope was reported to be $62 \mathrm{mv}$ per decade. $\mathrm{Zn}^{54}$ and $\mathrm{Cu}^{55}$ doped $\mathrm{Co}_{2} \mathrm{O}_{4}$ quantum dots ( $\mathrm{Zn}-\mathrm{Co}$ and $\mathrm{Cu}-\mathrm{Co}$ bimetallic oxide) on $\mathrm{N}$-doped carbon nanotubes prepared by hydrothermal methods were evaluated electrocatalytic water splitting activity. The former exhibited OER activity, while the latter exhibited both OER and HER. The electrolyte used for evaluating both catalysts was $0.1 \mathrm{M} \mathrm{KOH}$. For the $\mathrm{Zn}$ doped material ${ }^{54}$ a current density of $10 \mathrm{~mA} \mathrm{~cm}{ }^{-2}$ was observed at $1.65 \mathrm{~V}$ (OER), which was comparable to $\mathrm{IrO}_{2}$ on $\mathrm{N}$ doped carbon nanotubes. When the $\mathrm{Cu}-\mathrm{Co}_{2} \mathrm{O}_{4}$ based catalyst was evaluated for OER, a current density of $10 \mathrm{~mA} \mathrm{~cm}{ }^{-2}$ was observed at $0.7 \mathrm{~V}$ ( $v s$. SCE) while for HER a current density of $-5.5 \mathrm{~mA} \mathrm{~cm}{ }^{-2}$ was observed at $-0.7 \mathrm{~V}$ (vs. SCE).

An interesting report demonstrates in situ activation (by electrochemical oxidation reduction cycle-EORC) of stainless steel rust (mixture of $\mathrm{Ni}$ and Fe oxides) towards "low-cost, efficient, free-standing, and recoverable oxygen evolution electrodes." ${ }^{56}$ The process developed could "mimic and expedite" the corrosion process, and thus activate stainless steel as freestanding OER electrodes. The production of electrolyteaccessible $\mathrm{Fe} /(\mathrm{Ni})$ oxyhydroxides, as well as the high conductivity and mechanical stability, this electrode exhibited good OER performance including low overpotential, fast kinetics, and long-term durability. Another interesting aspect is that the minor degradation in current after long-term use could be handled in situ by an EORC. Mn doped Co tungstates, $\mathrm{Co}_{1-x^{-}}$ $\mathrm{Mn}_{x} \mathrm{WO}_{4}(x=0$ to 1$)$ were reported as bifunctional catalysts for OER and ORR. ${ }^{57}$ These nanocrystalline oxides with selective termination of low surface energy planes were synthesized by hydrothermal method. Substituting Mn enhances the catalytic activity leading to a negative shift in the $\mathrm{Co}^{2+} / \mathrm{Co}^{3+}$ redox wave and onset of the OER, indicating a strong $\mathrm{Mn}-\mathrm{Co}$ electronic interaction. $\mathrm{Co}_{0.5} \mathrm{Mn}_{0.5} \mathrm{WO}_{4}$ was found to be the most efficient catalyst in the series for both the OER and ORR with a combined overpotential of $0.89 \mathrm{~V}$. It exhibited an OER current density of 10 $\mathrm{mA} \mathrm{cm}{ }^{-2}$ at an overpotential of $400 \mathrm{mV}$. DFT calculations indicated that the substitution of $\mathrm{Mn}$ in $\mathrm{CoWO}_{4}$ elevates the $3 \mathrm{~d}$ metal d band center closer to the Fermi energy and hence eases the electron transfer to facilitate ORR and OER. Au/ $\mathrm{NiCo}_{2} \mathrm{O}_{4}$ arrays prepared hydrothermally were reported. ${ }^{58}$ These nanoarrays exhibited OER activity that was several folds higher than the well-known catalyst Ir/C (at $1.75 \mathrm{~V}$ vs. RHE). The $2 \mathrm{wt} \% \mathrm{Au}$ loading was the most active catalyst with a small Tafel slope (63 mV per decade) and an overpotential of $370 \mathrm{mV}$ to achieve a current density of $10 \mathrm{~mA} \mathrm{~cm}{ }^{-2}$. The activity of the catalysts with lower loadings $(1.5 \mathrm{wt} \% \mathrm{Au})$ and higher loadings (3.7 wt $\%$ $\mathrm{Au}$ ) catalysts were much lower. The catalysts show good stability in alkaline solution, making them suitable for water oxidation catalysis. It was hypothesized that the incorporation of Au acts as an electron sink and facilitates the oxidation of Co to +IV state, thus contributing to enhanced electrocatalytic activity. Au 

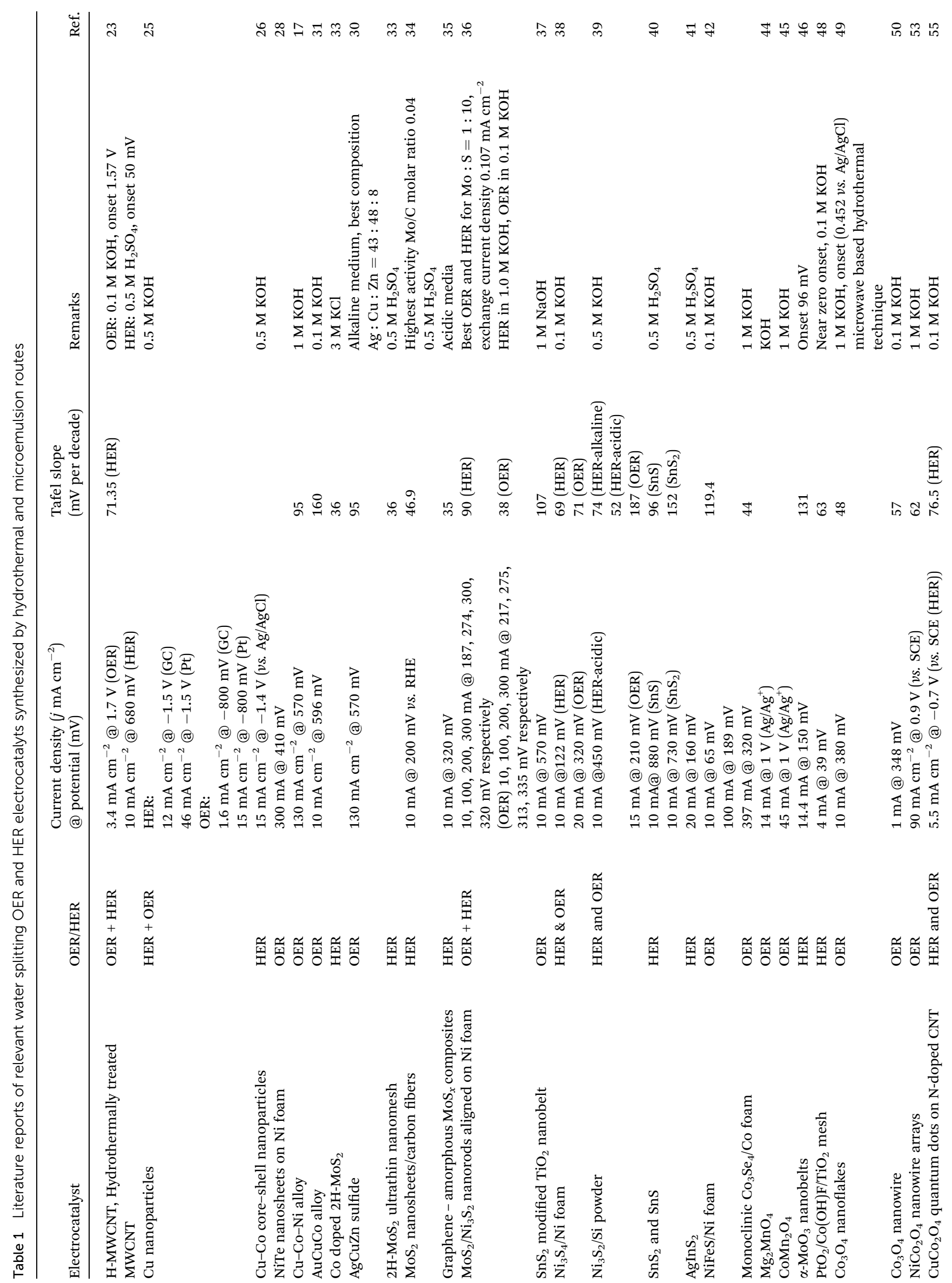


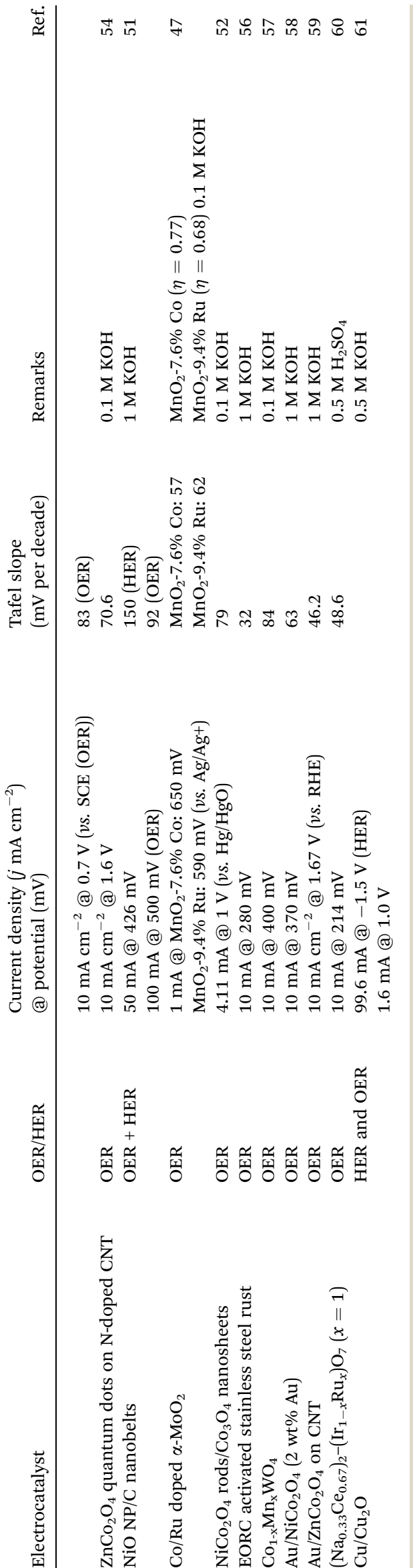

was also hypothesized to be helpful in the formation of the $\mathrm{OOH}$ intermediate that is highly unstable and decomposes to molecular $\mathrm{O}_{2}$ rapidly. A composite of spinel $\mathrm{ZnCo}_{2} \mathrm{O}_{4}$ and $\mathrm{Au}$ nanodots on CNT prepared by hydrothermal methods was evaluated for OER. ${ }^{59}$ The catalytic activity increases with increased Au loading and surpasses $\mathrm{IrO}_{2}$. A current density of 10 $\mathrm{mA} \mathrm{cm}{ }^{-2}$ was observed at $1.67 \mathrm{~V}$ (vs. RHE) while $97.8 \mathrm{~mA} \mathrm{~cm}^{-2}$ (vs. RHE) was observed at $1.8 \mathrm{~V}$ when $1 \mathrm{M} \mathrm{KOH}$ was used as an electrolyte. $\mathrm{RuO}_{2}$ is the most active catalyst for the OER but it is unstable and known to oxidize to $\mathrm{RuO}_{4}$. $\mathrm{IrO}_{2}$ is more stable, but has a lower activity. To address these shortcomings in the individual $\mathrm{Ru}$ and Ir based catalysts, a pyrochlore based solid solution $\left(\mathrm{Na}_{0.33} \mathrm{Ce}_{0.67}\right)_{2}-\left(\mathrm{Ir}_{1-x} \mathrm{Ru}_{x}\right)_{2} \mathrm{O}_{7}(0<x<1)$, containing $\mathrm{Ru}$ IV and Ir Iv at the B-site was evaluated as a catalyst for OER in acidic conditions. ${ }^{60}$ This catalyst was prepared by hydrothermal methods and the atomically mixed Ru and Ir as well as their nanocrystalline form allows effective fabrication of electrode coatings with improved charge density over the well-known $(\mathrm{Ru}, \mathrm{Ir}) \mathrm{O}_{2}$ catalysts. Using XANES spectroscopy an in situ study of the catalyst at the Ir L III and Ru K edges indicated that both $\mathrm{Ru}$ and Ir participate in the redox chemistry at OER conditions and that $\mathrm{Ru}$ is more active than Ir. Ru gets oxidized by almost one oxidation state at maximum applied potential, additionally, there was no evidence for presence of ruthenate or iridate in oxidation states of +6 or higher. The above studies can potentially be used for future catalyst development.

\subsection{Other miscellaneous materials}

Our group has reported the electrocatalytic activity of $\mathrm{Cu} / \mathrm{Cu}_{2} \mathrm{O}$ nanocomposites which were obtained from precursors synthesized by microemulsion methods. ${ }^{61}$ HER and OER were undertaken using these catalyst on glassy carbon as working electrodes in an alkaline solution. In HER experiments, these $\mathrm{Cu} / \mathrm{Cu}_{2} \mathrm{O}$ nanocomposites produce $\sim 8$ times higher current density than $\mathrm{Cu}$ and $\sim 4.5$ times higher than $\mathrm{Cu}_{2} \mathrm{O}$. In the case of OER studies, the current density is 36 times higher than $\mathrm{Cu}$ and 2.9 times higher than $\mathrm{Cu}_{2} \mathrm{O}$. The higher activity in the composites was attributed to the fast electron transfer rate at the interfaces in the nanocomposites. The electrocatalyst $(\mathrm{Cu} /$ $\mathrm{Cu}_{2} \mathrm{O}$ ) was stable over 50 cycles. Synthesis of $\mathrm{Ag}$ nanoparticles decorated hematite "nano-dendrite" electro-catalysts and its methanol assisted water splitting application has been investigated. ${ }^{62}$ The performance of catalyst synthesized by chemical precipitation was found to show better catalytic activity than the one prepared by hydrothermal methods. The observed enhancement in electro-catalytic properties was attributed to the synergistic effect of hematite dendrites as well as on the larger surface area of dendrite structure leading to higher loading of Ag NPs, however no concrete evidence to support this was provided. $\mathrm{Fe}$-doped $\mathrm{Ni}_{2} \mathrm{P}$ nanosheet arrays were reported as bifunctional catalysts (both HER and OER) for overall water splitting. ${ }^{63}$ These catalysts were synthesized by hydrothermal method followed by in situ phosphorization. The $\left(\mathrm{Ni}_{0.33} \mathrm{Fe}_{0.67}\right)_{2} \mathrm{P}$ exhibited good activity towards OER with a small overpotential of $\sim 214 \mathrm{mV}$ to achieve a current density of $50 \mathrm{~mA} \mathrm{~cm}{ }^{-2}$. However, there was strong evidence that active species for OER 
was an oxidized species $\mathrm{MO}_{x} / \mathrm{MOOH}(\mathrm{M}=\mathrm{Ni}, \mathrm{Fe}) / \mathrm{Fe}$-doped $\mathrm{Ni}_{2} \mathrm{P}$ rather than the phosphide species $\left(\mathrm{Ni}_{0.33} \mathrm{Fe}_{0.67}\right)_{2} \mathrm{P}$ itself. When used in an electrolyzer, a potential of $1.49 \mathrm{~V}$ was required to achieve a current density of $10 \mathrm{~mA} \mathrm{~cm} \mathrm{~cm}^{-2}$. Cobalt carbonate hydroxide supported on a carbon $(\mathrm{CCH} / \mathrm{C})$ catalyst synthesized via a hydrothermal method was reported to be an active catalyst for OER and ORR reactions. ${ }^{64}$ It was found that changing the reaction time leads to a phase transition from orthorhombic to monoclinic after $16 \mathrm{~h}$. The electrocatalytic properties of the composite were strongly dependent on the crystalline phase of the $\mathrm{CCH}$. $\mathrm{Ni}_{2} \mathrm{P}$ nanoparticles decorating the surface of $\mathrm{MoO}_{2} @ \mathrm{MoS}_{2}\left(\mathrm{Ni}_{2} \mathrm{P} / \mathrm{MoO}_{2} \mathrm{MoS}_{2}\right)$ were reported to be active for both HER and OER. ${ }^{65}$ The precursor, $\mathrm{NiMoO}_{4}$ wires growing on Ti foil were synthesized via a modified hydrothermal reaction. Incorporation of different classes of materials (viz oxides, phosphides and chalcogenides) in the same catalyst helps to achieve a unique multifunctional catalyst. Such techniques may be used for synthesis of various other multifunctional catalysts that are not limited to water splitting applications. Table 1 summarizes the water splitting activity of some of the catalysts that were synthesized by hydrothermal/microemulsion methods.

\section{Conclusions and current challenges}

Electrocatalysts synthesized by low temperature hydrothermal and microemulsion methods for water splitting applications were reviewed. Various categories of electrocatalysts like metal nanoparticles, binary and ternary alloys, metal oxides and metal chalcogenides have been discussed. The choice of electrocatalysts broadly corresponds to the classes of materials, synthesized by these above-mentioned techniques, that have been investigated by our group in the past. In general for the design of the catalysts, the different approaches adopted are (1) alloy different transition metals (2) synthesize composites with a large surface area, high crystallinity, large interlayer spacing and porous structure and (3) synthesize 3D structures supported usually on supports such as $\mathrm{Ni}$ foam, Cu foam etc. The shape and morphology of the nanoparticles was definitely found to influence the electrocatalytic properties. Thus, low temperature techniques like hydrothermal and microemulsion processes that allow control of morphology and similar physical properties are highly suitable for development of water splitting catalysts. However, one of the primary drawbacks for the use of microemulsion and hydrothermal based synthesis of electrocatalysts is the inherent difficulty in removal of the surfactants post synthesis. These surfactants are usually charged and may contain heteroatoms. Even though calcination results in removal of carbonaceous species, the role of other species, e.g. bromide ions in CTAB (a commonly used surfactant) that may interfere in electrocatalytic process is often ignored in literature. A more intensive approach to screen and address this issue is highly desirable. It is recommended that the scientific community use the available analytical tools to specifically look for probable interfering ions that may originate from surfactants.

Though some electrolysers have been developed where overall water splitting to produce $\mathrm{H}_{2}$ and $\mathrm{O}_{2}$ have been reported, an electrocatalyst which is bifunctional in terms of catalyzing both HER and OER in the same electrolyte is still a challenge. All the bifunctional catalysts (active for both HER and OER) reported here require more than $1.4 \mathrm{~V}$ to achieve a current density of $10 \mathrm{~mA} \mathrm{~cm}^{-2}$. At such high potentials, there are ample possibilities for a change in oxidation states of the constituent transition metals. It was seen, that in literature, a majority of the bifunctional catalysts, that catalyze both HER and OER have $\mathrm{Ni}$ as a constituent. It is noteworthy though that $\mathrm{Cu} / \mathrm{Cu}_{2} \mathrm{O}$ reported by us is bifunctional for both these reactions. Co based electrocatalysts are active towards OER reactions. There are reports of using multiple functionalities (viz oxides, phosphides and chalcogenides) in the same catalyst helps to achieve a unique multifunctional catalyst with favorable electrocatalytic properties. However, in such composites, the specific role of each component/functionality would be difficult to elucidate and thus a modular, bottom up approach with lesser number of components is recommended. Additionally, use of complex compositions may lead to waste management issues and the corresponding environmental ramifications. In general Co and $\mathrm{Ni}$ based catalysts are the best candidates for exploring overall water splitting applications. Though there have been reports of doping noble metals to enhance activity, industrial relevance based on economic viability should be considered. 2D transition metal dichalcogenides based on Mo are also potentially promising building blocks for such catalysts.

Additional challenges in the field are benchmarking, scalability and determination of the electrocatalytically active site. Benchmarking issues arise due to the use of non-standard conditions, electrolytes and their concentrations. As seen from Table 1, there is a lot of diversity in the choice of electrolyte for the HER and OER studies. Further, the role of the electrolyte and the possibility of interfering ions inherently present in them in often ignored. There are a few recent studies that ${ }^{66}$ have rigorously removed interfering ions (in this case Fe ions) from the electrolyte $(\mathrm{KOH})$, however, such practices are not usually carried out. Another point to note is that often, especially in some of the earlier studies, the role of impurities from the reaction vessel was ignored. Usually glass vessels were used and the highly basic conditions especially in OER reactions makes them prone to etching. The role of silica and the additives (that are etched out), were not thoroughly examined in these highly sensitive electrocatalytic experiments. Teflon vessels are now used as alternatives, however, still in these highly basic environments, the role of constituent ions such as fluoride in these is still an open question. Another aspect is the quality and the uniformity of the catalyst film on the electrode. Even though the proportion of the binder (e.g. Nafion) to the catalyst/support is usually reported, the actual process of physical deposition of the slurry on to the electrode is highly non-standard and the quality of the film depends on the "experimental hand" of the experimentalist. Often, this is also not reproducible, even in the same lab. Moreover, there is the issue of film degradation due to 
bubble formation resulting from the hydrogen and oxygen evolved. Adequate post catalysis studies are often not carried out to address the issue of stability and determination of the electrocatalytically active site.

Another major point that the current research is lacking is the scalability and stability. Preparation of catalyst loaded electrodes that can perform water splitting on an industrial scale for a reasonable number of cycles that would make it cost effective is still a challenge. As of 2016, the catalytic splitting of water to form hydrogen and molecular oxygen via electrolysis provides only $4 \%$ of the world's hydrogen. ${ }^{67}$ Thus, there is ample scope of development of better performing electrocatalysts for water splitting using hydrothermal and microemulsion techniques.

\section{Conflicts of interest}

There are no conflicts to declare.

\section{Acknowledgements}

AKG acknowledges the support of nanoscale research facility, IIT Delhi for part of the work described. AD acknowledges the support from the Council of Scientific and Industrial Research (CSIR-India) for financial support in form of SRA (Senior Research Associate - Pool Scheme) fellowship.

\section{References}

1 C. Burda, X. B. Chen, R. Narayanan and M. A. El-Sayed, Chem. Rev., 2005, 105, 1025-1102.

2 L. M. Liz-Marzan, Langmuir, 2006, 22, 32-41.

3 A. Gocalinska, M. Saba, F. Quochi, M. Marceddu, K. Szendrei, J. Gao, M. A. Loi, M. Yarema, R. Seyrkammer, W. Heiss, A. Mura and G. Bongiovanni, J. Phys. Chem. Lett., 2010, 1, 1149-1154.

4 M. Nasilowski, B. Mahler, E. Lhuillier, S. Ithurria and B. Dubertret, Chem. Rev., 2016, 116, 10934-10982.

5 G. Demazeau, J. Mater. Chem., 1999, 9, 15-18.

6 M. Nyman, M. A. Rodriguez, L. E. Shea-Rohwer, J. E. Martin and P. P. Provencio, J. Am. Chem. Soc., 2009, 131, 1165211653.

7 D. R. Modeshia and R. I. Walton, Chem. Soc. Rev., 2010, 39, 4303-4325.

8 R. E. Riman, W. L. Suchanek and M. M. Lencka, Ann. Chimie Sci. Materiaux., 2002, 27, 15-36.

9 J. L. West and N. J. Halas, Annu. Rev. Biomed. Eng., 2003, 5, 285-292.

10 X. C. Li, W. J. Zheng, G. H. He, R. Zhao and D. Liu, ACS Sustainable Chem. Eng., 2014, 2, 288-295.

11 M. P. Pileni, J. Phys. Chem., 1993, 97, 6961-6973.

12 M. P. Pileni, J. Exp. Nanosci., 2006, 1, 13-27.

13 M. P. Pileni, J. Phys. Chem. C, 2007, 111, 9019-9038.

14 M. P. Pileni, Langmuir, 1997, 13, 3266-3276.

15 C. Dhand, N. Dwivedi, X. J. Loh, A. N. Jie Ying, N. K. Verma, R. W. Beuerman, R. Lakshminarayanan and S. Ramakrishna, RSC Adv., 2015, 5, 105003-105037.
16 T. Ahmad and A. K. Ganguli, J. Am. Ceram. Soc., 2006, 89, 3140-3146.

17 S. Saha, K. V. Ramanujachary, S. E. Lofland and A. K. Ganguli, Mater. Res. Express, 2016, 3, 016501.

18 T. Ahmad and A. K. Ganguli, J. Mater. Res., 2004, 19, 29052912.

19 T. Ahmad, G. Kavitha, C. Narayana and A. K. Ganguli, J. Mater. Res., 2005, 20, 1415-1421.

20 S. Vaidya, T. Ahmad, S. Agarwal and A. K. Ganguli, J. Am. Ceram. Soc., 2007, 90, 863-869.

21 T. Ahmad, K. V. Ramanujachary, S. E. Lofland and A. K. Ganguli, J. Chem. Sci., 2006, 118, 513-518.

22 I. Roger, M. A. Shipman and M. D. Symes, Nat. Rev. Chem., 2017, 1, 0003.

23 B. H. R. Suryanto, S. Chen, J. J. Duan and C. Zhao, ACS Appl. Mater. Interfaces, 2016, 8, 35513-35522.

24 J. Ahmed, P. Trinh, A. M. Mugweru and A. K. Ganguli, Solid State Sci., 2011, 13, 855-861.

25 B. Kumar, S. Saha, M. Basu and A. K. Ganguli, J. Mater. Chem. A, 2013, 1, 4728-4735.

26 J. Ahmed, A. Ganguly, S. Saha, G. Gupta, P. Trinh, A. M. Mugweru, S. E. Lofland, K. V. Ramanujachary and A. K. Ganguli, J. Phys. Chem. C, 2011, 115, 14526-14533.

27 J. Ahmed, B. Kumar, A. M. Mugweru, P. Trinh, K. V. Ramanujachary, S. E. Lofland, Govind and A. K. Ganguli, J. Phys. Chem. C, 2010, 114, 18779-18784.

28 Y. Li and C. Zhao, Adv. Mater. Lett., 2017, 8, 916-921.

29 S. Saha, S. Vaidya, K. V. Ramanujachary, S. E. Lofland and A. K. Ganguli, Bull. Mater. Sci., 2016, 39, 433-436.

30 M. H. Xie, S. Q. Ai, J. Yang, Y. D. Yang, Y. H. Chen and Y. Jin, ACS Appl. Mater. Interfaces, 2015, 7, 17112-17121.

31 H. Gong, W. Zhang, F. Li and R. Yang, Electrochim. Acta, 2017, 252, 261-267.

32 K. Ojha, S. Saha, S. Banerjee and A. K. Ganguli, ACS Appl. Mater. Interfaces, 2017, 9, 19455-19461.

33 K. Zhang, H. J. Kim, J. T. Lee, G. W. Chang, X. Shi, W. Kim, M. Ma, K. J. Kong, J. M. Choi, M. S. Song and J. H. Park, Chemsuschem, 2014, 7, 2489-2495.

34 Y. Zhou, H. Q. Xiao, S. T. Wang, X. Pan, Z. J. Wang, C. H. An and J. Zhang, Mater. Chem. Phys., 2016, 183, 18-23.

35 J. Luxa, V. Mazanek, D. Bousa, D. Sedmidubsky, M. Pumera and Z. Sofer, ChemElectroChem, 2016, 3, 565-571.

36 N. Zhang, J. Lei, J. Xie, H. Huang and Y. Yu, RSC Adv., 2017, 7, 46286-46296.

37 M. Y. Li, H. Liu, T. Lv and M. Ding, J. Mater. Chem. A, 2018, 6, 3488-3499.

38 J.-T. Ren and Z.-Y. Yuan, ACS Sustainable Chem. Eng., 2017, 5, 7203-7210.

39 A. A. Ensafi, M. Jafari-Asl, A. Nabiyan and B. Rezaei, Energy, 2016, 116, 392-401.

40 X. Y. Chia, P. Lazar, Z. Sofer, J. Luxa and M. Pumera, J. Phys. Chem. C, 2016, 120, 24098-24111.

41 P. Saji, A. K. Ganguli, M. A. Bhat and P. P. Ingole, ChemPhysChem, 2016, 17, 1195-1203.

42 B. Dong, X. Zhao, G. Q. Han, X. Li, X. Shang, Y. R. Liu, W. H. Hu, Y. M. Chai, H. Zhao and C. G. Liu, J. Mater. Chem. A, 2016, 4, 13499-13508. 
43 W. Li, X. Gao, D. Xiong, F. Wei, W.-G. Song, J. Xu and L. Liu, Adv. Energy Mater., 2017, 7, 1602579.

44 N. Garg, Menaka, K. V. Ramanujachary, S. E. Lofland and A. K. Ganguli, J. Solid State Chem., 2013, 197, 392-397.

45 N. Garg, M. Mishra, Govind and A. K. Ganguli, RSC Adv., 2015, 5, 84988.

46 H. Sinaim, D. J. Ham, J. S. Lee, A. Phuruangrat, S. Thongtem and T. Thongtem, J. Alloys Compd., 2012, 516, 172-178.

47 M. Luebke, A. Sumboja, L. McCafferty, C. F. Armer, A. D. Handoko, Y. Du, K. McColl, F. Cora, D. Brett, Z. Liu and J. A. Darr, ChemistrySelect, 2018, 3, 2613-2622.

48 Z. Q. Wang, Z. Liu, G. Du, A. M. Asiri, L. Wang, X. N. Li, H. J. Wang, X. P. Sun, L. Chen and Q. J. Zhang, Chem. Commun., 2018, 54, 810-813.

49 S. Chen, Y. Zhao, B. Sun, Z. Ao, X. Xie, Y. Wei and G. Wang, ACS Appl. Mater. Interfaces, 2015, 7, 3306-3313.

$50 \mathrm{~S} . \mathrm{Xu}, \mathrm{J}$. Tong, Y. Liu, W. Hu, G. Zhang and Q. Xia, J. Renewable Sustainable Energy, 2016, 8, 044703.

51 Q. Dong, C. Sun, Z. Dai, X. Zang and X. Dong, ChemCatChem, 2016, 8, 3484-3489.

52 P. Sennu, H. S. Park, K. U. Park, V. Aravindan, K. S. Nahm and Y.-S. Lee, J. Catal., 2017, 349, 175-182.

53 Y.-Z. Su, Q.-Z. Xu, G.-F. Chen, H. Cheng, N. Li and Z.-Q. Liu, Electrochim. Acta, 2015, 174, 1216-1224.

54 L. Zhao-Qing, C. Hui, L. Nan, M. T. Yi and S. Yu-Zhi, Adv. Mater., 2016, 28, 3777-3784.

55 C. Hui, L. Mei-Ling, S. Chang-Yuan, L. Nan and L. ZhaoQing, Adv. Funct. Mater., 2017, 27, 1701833.
56 H. Zhong, J. Wang, F. Meng and X. Zhang, Angew. Chem., Int. Ed., 2016, 55, 9937-9941.

57 G. Karkera, T. Sarkar, M. D. Bharadwaj and A. S. Prakash, ChemCatChem, 2017, 9, 3681-3690.

58 X. J. Liu, J. F. Liu, Y. P. Li, Y. J. Li and X. M. Sun, ChemCatChem, 2014, 6, 2501-2506.

59 H. Cheng, C.-Y. Su, Z.-Y. Tan, S.-Z. Tai and Z.-Q. Liu, J. Power Sources, 2017, 357, 1-10.

60 K. Sardar, E. Petrucco, C. I. Hiley, J. D. B. Sharman, P. P. Wells, A. E. Russell, R. J. Kashtiban, J. Sloan and R. I. Walton, Angew. Chem., Int. Ed., 2014, 53, 10960-10964.

61 B. Kumar, S. Saha, K. Ojha and A. K. Ganguli, Mater. Res. Bull., 2015, 64, 283-287.

62 P. Chaudhary and P. P. Ingole, Int. J. Hydrogen Energy, 2018, 43, 1344-1354.

63 Y. Li, H. Zhang, M. Jiang, Q. Zhang, P. He and X. Sun, Adv. Funct. Mater., 2017, 27, 1702513.

64 Y. Wang, W. Ding, S. Chen, Y. Nie, K. Xiong and Z. Wei, Chem. Commun., 2014, 50, 15529-15532.

65 Y. Wang, T. Williams, T. Gengenbach, B. Kong, D. Zhao, H. Wang and C. Selomulya, Nanoscale, 2017, 9, 17349-17356.

66 M. Burke, M. G. Kast, L. Trotochaud, A. Smith and S. W. Boettcher, J. Am. Chem. Soc., 2015, 137, 3638-3648.

67 Engineers Ireland, Electrochemical water splitting: a new dawn for the hydrogen economy, http:// www.engineersjournal.ie/2016/07/12/electrochemical-watersplitting-hydrogen-economy/. 